\title{
BMJ Open Association of dispositional optimism with Life's Simple 7's Cardiovascular Health Index: results from the Hispanic Community Health Study/Study of Latinos (HCHS/SOL) Sociocultural Ancillary Study (SCAS)
}

Rosalba Hernandez, ${ }^{1}$ Hector M González, ${ }^{2}$ Wassim Tarraf, ${ }^{3}$ Judith T Moskowitz, ${ }^{4}$ Mercedes R Carnethon, ${ }^{5}$ Linda C Gallo, ${ }^{6}$ Frank J Penedo, ${ }^{4}$ Carmen R Isasi, ${ }^{7}$ John Manuel Ruiz, ${ }^{8}$ William Arguelles, ${ }^{9,10}$ Christina Buelna, ${ }^{11}$ Sonia Davis, ${ }^{12}$ Franklyn Gonzalez, ${ }^{12}$ Jessica L McCurley, ${ }^{13}$ Donghong Wu, ${ }^{14}$ Martha L Daviglus ${ }^{5,14}$

To cite: Hernandez $\mathrm{R}$, González HM, Tarraf W, et al. Association of dispositional optimism with Life's Simple 7's Cardiovascular Health Index: results from the Hispanic Community Health Study/ Study of Latinos (HCHS/ SOL) Sociocultural Ancillary Study (SCAS). BMJ Open 2018;8:e019434. doi:10.1136/ bmjopen-2017-019434

- Prepublication history for this paper is available online. To view these files, please visit the journal online (http://dx.doi. org/10.1136/bmjopen-2017019434).

Received 5 September 2017 Revised 24 October 2017 Accepted 3 November 2017

Check for updates

For numbered affiliations see end of article.

Correspondence to Dr Rosalba Hernandez; rherna17@illinois.edu

\section{ABSTRACT}

Objectives Mounting evidence links positive psychological functioning to restorative health processes and favourable medical outcomes. However, very little is known about the relationship between optimism, an indicator of psychological functioning and the American Heart Association (AHA)-defined concept of cardiovascular health (CVH), particularly in Hispanics/ Latinos of diverse backgrounds. To address limitations of existing literature, this study investigated the association between dispositional optimism and CVH in a heterogeneous sample of Hispanics/Latinos residing in the USA.

Design Cross-sectional study.

Participants and setting Data were analysed from 4919 adults ages 18-75 of the Hispanic Community Health Study/Study of Latinos parent study and the Sociocultural Ancillary Study.

Main outcome measures Optimism was assessed using the 6-item Life Orientation Test-Revised (range from 6 to 30). AHA classification standards were used to derive an additive CVH score with operationalisation of indicators as Ideal, Intermediate and Poor. The overall CVH score included indicators of diet, body mass index, physical activity, cholesterol, blood pressure, fasting glucose and smoking status. Multivariate linear and logistic regressions were used to examine associations of optimism with CVH (Life's Simple 7), after adjusting for sociodemographic factors and depressive symptoms.

Results Each increase in the optimism total score was associated with a greater CVH score $(\beta=0.03$ per unit increase, $95 \% \mathrm{Cl} 0.01$ to 0.05 ). When modelling tertiles of optimism, participants with moderate ( $\beta=0.24$ to $95 \% \mathrm{Cl} 0.06$ to 0.42$)$ and high $(\beta=0.12$, $95 \% \mathrm{Cl} 0.01$ to 0.24 ) levels of optimism displayed greater CVH scores when compared with their least optimistic peers.
Strengths and limitations of this study

- This is one of the few studies to examine the link between dispositional optimism and the seminal concept of cardiovascular health (CVH) which focuses on maintenance and promotion of favourable behavioural health practices and biological attributes.

- The strength of this study is exploration of the association between emotional well-being and cardiac-related health in a large heterogeneous sample of Hispanic/Latino adults that afforded the power to examine effect modification by country of origin.

- Our study contributes to existing literature and suggests that dispositional optimism may be a novel therapeutic target for consideration when attempting to promote CVH among Hispanic/Latino adults, regardless of age, sex or Hispanic/Latino country of origin.

- We acknowledge the inherent limitation associated with cross-sectional designs and endorse longitudinal studies and randomised trials to ascertain hypotheses regarding causation and temporality of the association.

- Because dietary intake and physical activity were assessed through self-report, measurement error and misclassification bias were possible.

Conclusion This study offers preliminary evidence for an association between optimism and CVH in a large heterogeneous group of Hispanic/Latino adults. Our study adds scientific knowledge of psychological assets that may promote CVH and suggests a novel therapeutic target for consideration. Future studies are needed to explore causality and potential mechanism underlying the relationship between positive emotion and heart health. 


\section{INTRODUCTION}

The American Heart Association (AHA) has called for the inclusion of depression as a cardiovascular disease (CVD) risk factor, joining the ranks of traditional markers such as diabetes and hypertension, to underscore the impact of emotional well-being on cardiovascular health $(\mathrm{CVH}) .^{1}$ In parallel to the AHA's recommendation, the field of health and medicine has broadened its focus from the deleterious health effects of negative psychological constructs to a positive orientation that focuses on psychological attributes such as happiness, optimism and life purpose. ${ }^{23}$ Dispositional optimism, that is, the overarching expectation that positive things will occur in the future, is highly correlated with positive emotion and has emerged as a well-being indicator of particular salience for cardiac-related health. ${ }^{45}$ Although well-being domains are often correlated, optimism largely focuses on expectations for a favourable future and less on present moment subjective feelings, and it has garnered the largest body of evidence linking it to superior cardiac health, as shown by longitudinal studies ${ }^{6}$ and comprehensive meta-analyses. ${ }^{7}$ Independent of major CVD risk factors, dispositional optimism consistently emerges as a cardioprotective factor and is independently associated with favourable lipid profiles, ${ }^{8}$ reduced circulation of inflammatory markers, lower rates of hospital readmission after coronary artery bypass graft surgery, reduced all-cause and cardiac-related mortality ${ }^{910}$ and reduced odds for incident heart disease. ${ }^{11}$

Notably, a majority of studies on the cardioprotective influence of positive emotion focus on disease states, deficits and disability and failure to consider the seminal concept of CVH (ie, Life's Simple 7 (LS7)) which focuses on maintenance and promotion of favourable behavioural health practices and biological attributes. Favourable profiles of $\mathrm{CVH}$ are related to increased longevity, better cognitive functioning, compressed extant morbidity, greater CVD-free survival and reduced odds for incident acute cardiovascular events. ${ }^{12}$ Few studies have explored the relationship between optimism and positive CVH (LS7). ${ }^{13-15}$ In participants of the Multi-Ethnic Study of Atherosclerosis (MESA), which included a substantial number of Hispanic/ Latino adults, Hernandez et $\mathrm{l}^{14}$ found two times greater odds of having ideal CVH for adults in the highest quartile of optimism when compared with least optimistic peers, independent of plausible confounds. ${ }^{14}$ However, that study was not able to explore whether the association was present among Hispanics/Latinos of diverse backgrounds as MESA was predominantly composed of Hispanics/Latinos endorsing Mexican ancestry. Major theories informing the concept of psychological wellbeing in the USA draw heavily from Western-centric perspectives, which often neglect culturally bound frameworks. Indeed, cultural attributes could result in divergent conceptions of psychological attributes and subsequent non-equivalent associations with physical health.

To address limitations of existing literature, this study investigated the association between dispositional optimism and CVH in a heterogeneous sample of Hispanics/Latinos residing in the USA. We hypothesised that greater levels of optimism would be associated with more favourable $\mathrm{CVH}$ profiles with variance in the stability and magnitude of the association based on important sociodemographic factors.

\section{METHODS}

\section{Study population and data source}

Participants include Hispanic/Latino adults (aged 18-74 years; $n=5313$ ) enrolled in the Hispanic Community Health Study/Study of Latinos (HCHS/SOL) parent study and the Sociocultural Ancillary Study (SCAS). ${ }^{16} 17$ The HCHS/SOL is a large community-based multicentre cohort study that seeks to identify risk and protective factors for chronic disorders and to quantify patterns of morbidity and mortality in a heterogeneous group of Hispanic/Latino adults. Details of the recruitment and study protocol for HCHS/SOL ${ }^{16}$ and $\mathrm{SCAS}^{17}$ have been previously published. Briefly, study enrolment for HCHS/SOL occurred from 2008 to 2011 across four US regions (New York, Illinois, California and Florida) and included a total of 16415 adults between the ages of 18 and 74 years. The sample included adults reporting heritage from Mexico, Cuba, Puerto Rico, the Dominican Republic and Central or South America. Participants were selected using a two-stage area household probability sampling approach. The SCAS consists of a subsample of 5313 participants in the original HSHS/SOL cohort with the aim of exploring socioeconomic, cultural and psychosocial influences on health. SCAS assessments were completed by enrollees within 9 months of their initial baseline HCHS/SOL examination. Studies were approved by the Institutional Review Boards at the data coordinating centre and at each field centre where all subjects gave written consent.

The final sample for this study was limited to participants with available scores for measures of optimism and across indicators needed to compute the CVH score (ie, $\mathrm{n}=4919$ or $92.6 \%$ of the original SCAS sample). Participants excluded from analysis were less likely to be married or living with a partner and they reported inferior emotional $(\mathrm{P}<0.01)$ and physical health $(\mathrm{P}<0.01)$; no significant differences were evident by age $(\mathrm{P}=0.06)$ or income $(\mathrm{P}=0.08)$.

\section{Study measures}

Dispositional optimism

Collected as part of SCAS, the Life Orientation Test-Revised (LOT-R) was used to assess dispositional optimism. ${ }^{18}$ The LOT-R is a self-administered questionnaire with possible scores ranging from 6 (least optimistic) to 30 (most optimistic) and includes items such as, 'In uncertain times, 
Table 1 Definitions for the three-category indicator of cardiovascular health, per American Heart Association (AHA) specifications

\begin{tabular}{|c|c|c|c|}
\hline $\begin{array}{l}\text { Cardiovascular health-LS7 } \\
\text { indicators }\end{array}$ & Poor & Intermediate & Ideal \\
\hline \multicolumn{4}{|l|}{ Three health behaviours } \\
\hline Physical activity & None & $\begin{array}{l}1-149 \mathrm{~min} / \text { week moderate, or } 1-74 \mathrm{~min} / \\
\text { week vigorous, or } 1-149 \mathrm{~min} / \text { week } \\
\text { combined intensity }\end{array}$ & $\begin{array}{l}\geq 150 \mathrm{~min} / \text { week moderate, } \\
\text { or } \geq 75 \mathrm{~min} / \text { week vigorous, } \\
\text { or } \geq 150 \mathrm{~min} / \text { week } \\
\text { combined intensity }\end{array}$ \\
\hline Smoking & Current smoker & Former smoker who quit $\leq 12$ months ago & $\begin{array}{l}\text { Never smoked or } \\
\text { quit }>12 \text { months ago }\end{array}$ \\
\hline \multicolumn{4}{|l|}{ Four biological markers } \\
\hline BMI $\left(\mathrm{kg} / \mathrm{m}^{2}\right)$ & $\geq 30$ or $<18.5$ & $\geq 25$ to $<30$ & $<25$ but $\geq 18.5$ \\
\hline Blood pressure (mm Hg) & $\geq 140$ or $\geq 90 \mathrm{~mm} \mathrm{Hg}$ & $120-193 / 80-89 \mathrm{~mm} \mathrm{Hg}$ or treated to control & $<120 /<80 \mathrm{~mm} \mathrm{Hg}$ \\
\hline
\end{tabular}

*AHA Diet score includes five criteria: $\geq 4.5$ servings/day fruits and vegetables; $\geq 7$ oz servings/week fish; $\geq 3$ servings/day grain; $\leq 4.5$ servings/ week sweetened beverages; and $<1500 \mathrm{mg} /$ day sodium. BMI, body mass index; LS7, Life's Simple 7.

I usually expect the best.' The scale includes three positively worded items, three negatively worded items and three filler items (excluded from analysis), all rated on a 5-point Likert-type scale with response options ranging from I agree a lot to I disagree a lot. As recommended by scale developers, main analyses treated the full-scale LOT-R as a unidimensional scale pooling all six items to generate a composite score of total optimism. In addition to treating the optimism score as a continuous measure, tertiles were created based on the distribution of scores to test for possible threshold effects (as clinically based cut-offs are unavailable). Given debate on the factorial structure of the LOT-R, second-order analyses examined optimism and pessimism subscales by parsing negatively and positively worded items.

\section{Cardiovascular health: Life's Simple 7}

AHA specifications ${ }^{19}$ were used to define and operationalise indicators of CVH for smoking, diet, physical activity, body mass index (BMI), fasting plasma glucose, serum cholesterol and blood pressure. ${ }^{20}$ Indicators were coded using a three-category scheme of Poor (0), Intermediate (1) or Ideal (2) (see table 1). A total CVH score was computed by summing the seven indicators to derive a score ranging from 0 to 14 , with higher scores indicative of better CVH. ${ }^{19}$ An LS7 index (range 0-7) was also considered based on the sum of Ideal criteria. Finally, a dichotomous LS7 CVH cut-point ( $\geq 4$ Ideal indicators) was generated that has been previously linked with cardioprotection and reduced 20-year incidence of coronary heart disease. ${ }^{121-23}$

Protocols to capture CVH (ie, LS7) indicators have been described in depth elsewhere. ${ }^{16}$ Briefly, former and current smoking status was self-reported. Two 24-hour dietary recalls were used to evaluate dietary intake and considered five food categories of fruits/vegetables, fish, grains, sweetened beverages and sodium. Physical activity was determined through self-report using a modified version of the Global Physical Activity Questionnaire which taps into domains related to work, transport and leisure. ${ }^{2425}$ The biomarkers considered were BMI, total cholesterol, fasting blood glucose and blood pressure. BMI, measured as $\mathrm{kg} / \mathrm{cm}^{2}$, was calculated from staff-ascertained measures of weight (nearest $0.1 \mathrm{~kg}$ ) and height (nearest centimetre). After a 12-hour fasting, blood was drawn to obtain lipid profiles and fasting glucose values. Total cholesterol was measured using a cholesterol oxidase enzymatic method while the hexokinase enzymatic method was employed when measuring fasting blood glucose (Roche Diagnostics, Roche Diagnostics, Indianapolis, Indiana, USA). Three systolic and diastolic blood pressure readings were taken with participants in a seated position; mean values were obtained by averaging across all three readings. Self-reported medication use was considered when identifying those with pre-existing diabetes, hypercholesterolaemia and hypertension. Information across $\mathrm{CVH}$ indicators was obtained during the HCHS/SOL baseline assessment (2008-2011).

\section{Covariates}

Covariates included baseline age, sex (male, female), educational attainment (less than high school (HS), HS graduate/general education degree, greater than HS), income ( $<$ US $\$ 20,000$, US $\$ 20000$ to US $\$ 50000$, $>$ US $\$ 50$ 000, not reported), marital status (married/ living as married/living with a partner), healthcare insurance status (has health insurance, does not have health insurance), nativity/immigration status, language preference for HCHS/SOL interview (English vs Spanish) and Hispanic/Latino heritage group (Mexican, Cuban, 
Puerto Rican, Dominican, Central American, South American or other). In addition, self-reported physical health was considered as measured using the Physical Health Composite Scale of the 12-item Short Form Health Survey. ${ }^{26}$ Finally, prevalent coronary heart disease (CHD) at baseline and depressive symptoms (Centre for Epidemiologic Studies-Depression Scale ${ }^{27}$ were considered as covariates.

\section{Statistical methods}

Complex survey-specific procedures were performed across analyses to properly account for sample weights and the two-stage sampling design involving clustering and stratification procedures. ${ }^{1628}$ Descriptive characteristics are presented by tertiles of optimism. Group differences in participant characteristics across optimism tertiles were examined using an F-test or $\chi^{2}$-test as appropriate.

The association of optimism and CVH was examined using multivariate linear and logistic regression. Observations with missing data were excluded from analyses. The first set of regression models treated the independent variable as a continuous score ranging from 6 (least optimistic) to 30 (most optimistic) with modelling of a one-unit increase in optimism. Three separate models were constructed using three CVH scoring methods, that is, continuous (0-14 and 0-7) and dichotomous ( $\geq 4$ Ideal). Model 1 was unadjusted. Model 2 adjusted for age, sex, Hispanic/ Latino heritage, marital status, education, income, insurance status, nativity/immigration status and language of interview. Model 3 was additionally adjusted for self-rated physical health, prevalent CHD and depressive symptoms. Analyses were replicated with treatment of optimism as a categorical (tertiled) variable to assess possible discontinuous effects (low: $\leq 21$; moderate: 22 to $\leq 25$; high: $\geq 26$ ) with the lowest tertile of optimism serving as the reference category. Sensitivity analyses testing the association of optimism and CVH were performed using logistic quantile regression to account for possible characterisation of $\mathrm{CVH}$ as a bounded outcome. ${ }^{29}$

Effect modification was also explored to inform whether stratified analyses were warranted through inclusion of interaction terms testing the stability of adjusted associations between optimism and cardiovascular by age, sex and Hispanic/Latino background. Finally, secondorder analyses treated the LOT-R as a bidimensional scale with subscales of optimism and pessimism. Data analysis was conducted using SAS V.9.4. Parameter estimators of logistic quantile regression models and corresponding 95\% CIs were estimated using bootstrap simulations.

\section{RESULTS}

\section{Sample characteristics}

Table 2 presents participant characteristics according to level of optimism. Participants ranged in age from 18 to 74 years $(\mathrm{M}=42.3, \mathrm{SE}=0.4)$ with an approximate equal distribution by sex (54.6\% female). The largest Hispanic/Latino group comprised those of Mexican heritage $(37.4 \%)$ followed by those identified as Cuban (20.1\%), Puerto Rican (15.5\%), Dominican (11.5\%) and Central (7.4\%) and South American (4.7\%). Overall, $32.5 \%$ had less than a HS education and $46.4 \%$ reported an annual income below US $\$ 20000$. P values presenting between-subject differences across demographic factors are presented by tertile of optimism (table 2). Participants categorised as most optimistic tended to be older, reported being married and/or living with a partner and displayed greater socioeconomic standing as quantified by education and income. Differences in optimism were also evident by heritage group. Specifically, a greater proportion of Cuban and Central American participants were in the highest optimism tertile as opposed to the lowest tertile, whereas this finding was reversed for those of Mexican and Puerto Rican heritage. Compared with their less optimistic peers, more favourable profiles among the most optimistic were evident for symptoms of depression. Table 2 also presents the bivariate association between tertiles of optimism and extant number of Ideal $\mathrm{CVH}$ indicators. Results suggest a gradient in the distribution of Ideal LS7 criteria across tertiles of optimism with lower levels of optimism associated with fewer Ideal LS7.

\section{Association between optimism and CVH}

As effect modification was not evident by sex, age or Hispanic/Latino heritage group, table 3 presents pooled estimates for the associations of optimism and $\mathrm{CVH}$ with optimism treated as a continuous variable. In the unadjusted model, each unit increase in optimism was associated with a higher CVH score $(\beta=0.03,95 \%$ CI 0.01 to 0.06$)$. This relationship was robust to inclusion of covariates capturing demographic factors, health status and depressive symptoms. Similar findings were evident across the distinct operationalisation of CVH ( $0-7$ continuous index and 4+Ideal LS7s). For instance, each unit increase in optimism was related to $3 \%$ higher odds of meeting Ideal criteria across four or more CVH metrics (4+Ideal LS7s; OR 1.03, 95\% CI 1.01 to 1.06 ).

Table 4 presents the $\beta$ estimates and associated CIs for the association of optimism tertiles and $\mathrm{CVH}$, with the least optimistic group serving as the referent category. In multivariate models and when compared with least optimistic peers, persons with mid and high levels of optimism displayed greater $\mathrm{CVH}$ scores when treating $\mathrm{CVH}$ as a continuous indicator (0-14 and 0-7 continuous indices). Specifically, mid levels of optimism were associated with greater CVH scores using the $0-14$ scaling ( $\beta=0.24,95 \%$ CI 0.06 to 0.42 ) and high levels of optimism were related to greater CVH on the $0-7$ scale $(\beta=0.1295$, \% CI 0.01 to 0.24$)$. Associations were robust to adjustment for demographic factors, physical health status and depressive symptoms. Statistically significant findings were not replicated when treating $\mathrm{CVH}$ as a dichotomous outcome (ie, presence of $\geq 4$ Ideal indicators vs $<4$ ).

Sensitivity analysis using logistic quantile regression resulted in identical findings as above. Finally, second-order analyses were conducted to explore the 


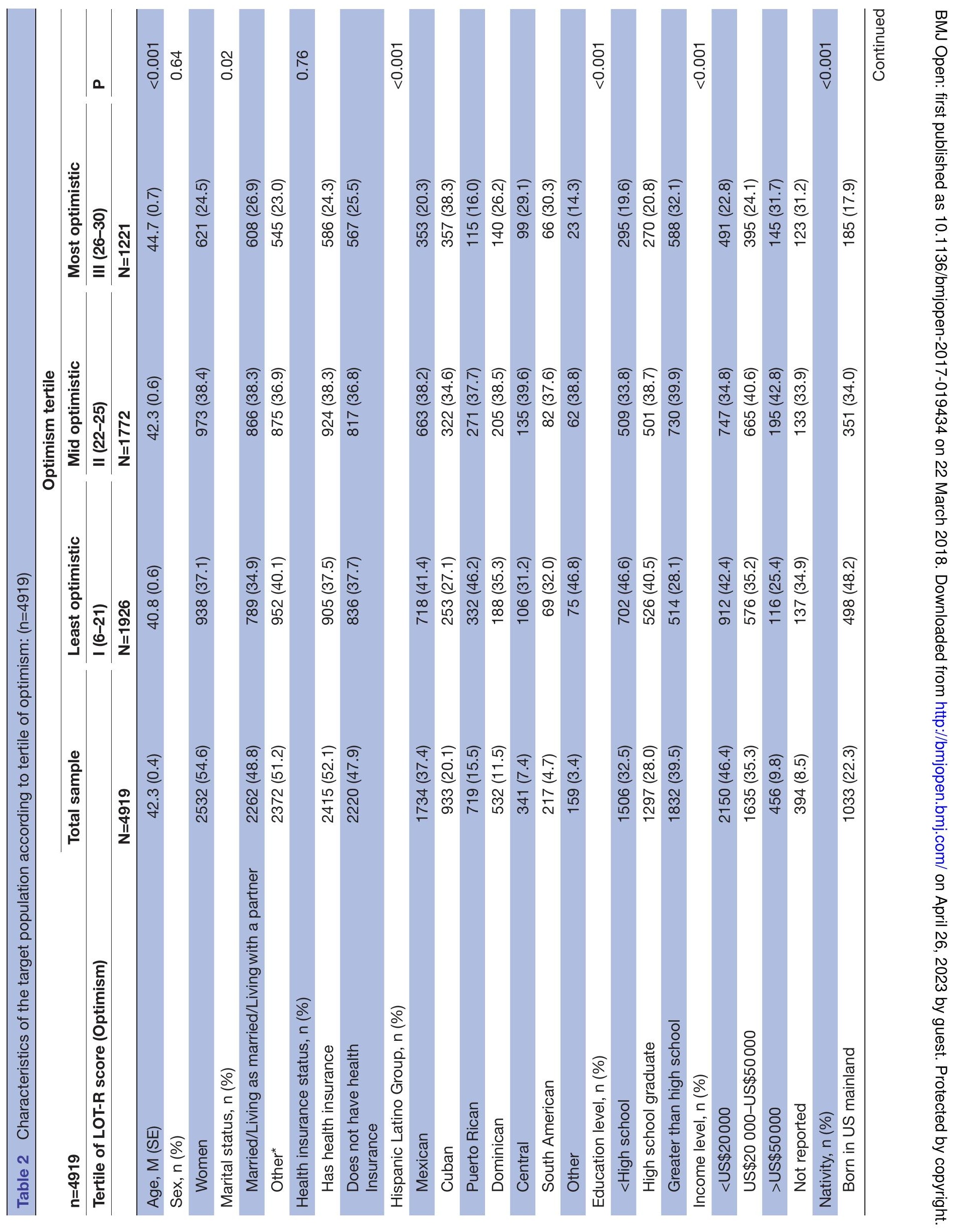




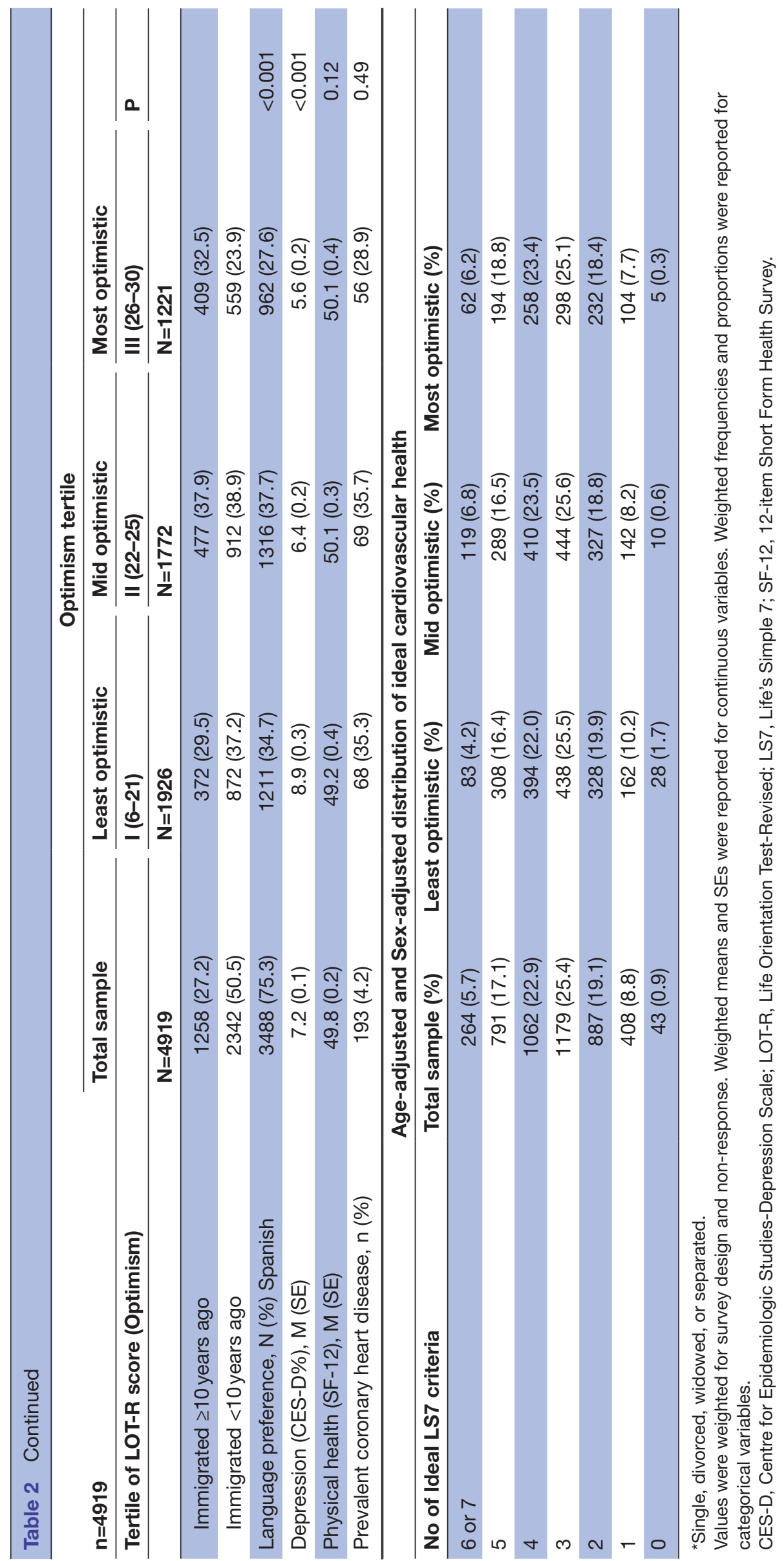


Table 3 Regression models of the association between one unit increase in optimism and cardiovascular health (continuous and categorical) $(\mathrm{N}=4919)$

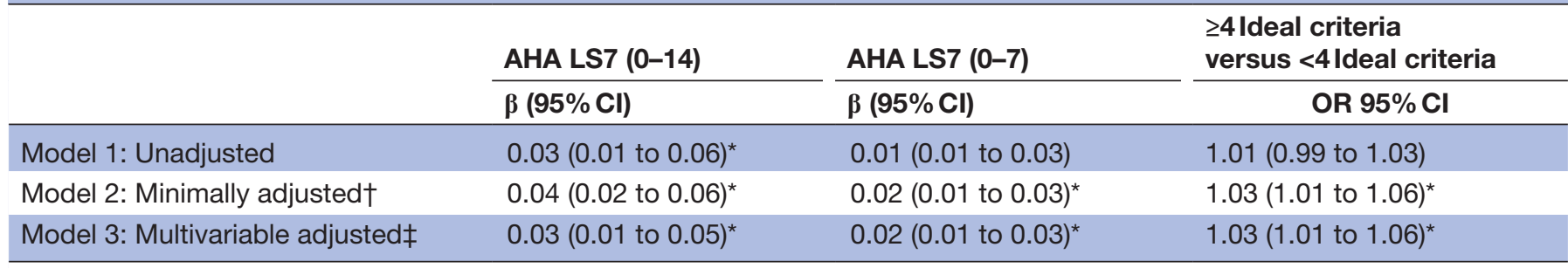

${ }^{*} \mathrm{P}<0.05$.

†Adjusted for age, sex, Hispanic/Latino national origin, marital status, education, income, insurance status, nativity/immigration status and language of interview.

$\ddagger$ Adjusted for age, sex, Hispanic/Latino national origin, marital status, education, income, insurance status, nativity/immigration status, language of interview, depressive symptoms (Centre for Epidemiologic Studies-Depression Scale), Physical Health Component of the 12-item Short Form Health Survey and prevalent coronary heart disease.

AHA, American Heart Association; LS7, Life's Simple 7.

association of the optimism and pessimism subscales with CVH. Associations of the LOT-R and CVH were largely driven by the negatively worded items $(\beta=-0.03$, $\mathrm{P}=0.03)$, this is in contrast to non-significant findings for the three-item cluster of positively worded items $(\beta=0.01, P=0.45)$ (not shown).

\section{DISCUSSION}

Limited research has investigated plausible psychosocial antecedents of favourable CVH despite its association with reduced risk of cardiac and all-cause mortality. In the first study to include Hispanics/Latinos of diverse heritage backgrounds, we found that higher optimism scores were associated with better CVH with no evidence of effect modification by age, sex or country of origin. Conversely, few Hispanics/Latinos with low optimism met the criteria for Ideal CVH. Our findings were not markedly affected by other factors measured that serve as plausible confounders (eg, prevalent CHD, depressive symptoms). In order to achieve the AHA's Strategic Impact Goals of improved $\mathrm{CVH}$ and reduced cardiac-related mortality by $20 \%$ by $2020,{ }^{19}$ novel and calculated strategies targeting vulnerable populations are needed to promote and maintain Ideal CVH across the lifespan.

Table 4 Cross-sectional associations of tertiles of optimism and cardiovascular health (LS7) ( $N=4919)$

\begin{tabular}{|c|c|c|c|}
\hline \multirow[b]{4}{*}{ Six-item LOT-R } & \multicolumn{3}{|c|}{ Cardiovascular health } \\
\hline & \multicolumn{2}{|c|}{ Continuous indicator(s) } & \multirow{2}{*}{$\begin{array}{l}\text { Dichotomous indicator } \\
\geq 4 \text { Ideal versus }<4 \text { Ideal }\end{array}$} \\
\hline & AHA LS7 (0-14) & AHA LS7 (0-7) & \\
\hline & $\beta(95 \% \mathrm{Cl})$ & $\beta(95 \% \mathrm{Cl})$ & OR $(95 \% \mathrm{Cl})$ \\
\hline Tertile 1-Lowest (reference group) & 1.0 (reference) & 1.0 (reference) & 1.0 (reference) \\
\hline \multicolumn{4}{|l|}{ M1: Unadjusted } \\
\hline Tertile II & $0.33(0.12 \text { to } 0.55)^{*}$ & $0.11(-0.02$ to 0.23$)$ & $1.08(0.89$ to 1.31$)$ \\
\hline Tertile III-Highest & $0.14(-0.09$ to 0.36$)$ & $0.03(-0.11$ to 0.17$)$ & $0.98(0.79$ to 1.21$)$ \\
\hline \multicolumn{4}{|l|}{ M2: Minimally adjusted $\dagger$} \\
\hline Tertile II & $0.28(0.09 \text { to } 0.47)^{\star}$ & $0.11(0.003 \text { to } 0.21)^{*}$ & $1.11(0.90$ to 1.36$)$ \\
\hline Tertile III-Highest & $0.23(0.03 \text { to } 0.43)^{*}$ & $0.14(0.03 \text { to } 0.26)^{*}$ & $1.15(0.91$ to 1.44$)$ \\
\hline \multicolumn{4}{|l|}{ M3: Multivariable adjusted $\ddagger$} \\
\hline Tertile II & $0.24(0.06 \text { to } 0.42)^{\star}$ & $0.09(-0.01$ to 0.20$)$ & 1.09 (0.89 to 1.34$)$ \\
\hline Tertile III-Highest & $0.18(-0.02$ to 0.38$)$ & $0.12(0.01 \text { to } 0.24)^{\star}$ & 1.13 (0.89 to 1.42$)$ \\
\hline
\end{tabular}

Tertiles range from lowest (I) to highest (III) for the LOT-R measure, with tertile III corresponding to the highest levels of optimism for the full six-item LOT-R measure.

${ }^{*} \mathrm{P}<0.05$.

†Adjusted for age, sex, Hispanic/Latino national origin, marital status, education, income, insurance status, nativity/immigration status and language of interview.

$\ddagger$ Adjusted for age, sex, Hispanic/Latino national origin, marital status, education, income, insurance status, nativity/immigration status, language of interview, depressive symptoms (Centre for Epidemiologic Studies-Depression Scale), Physical Health Component of the 12-item Short Form Health Survey and prevalent coronary heart disease.

AHA, American Heart Association; LOT-R, Life Orientation Test-Revised; LS7, Life's Simple 7. 
Our results are consistent with previous studies demonstrating the protective cardiac-related health effects of psychological well-being. ${ }^{14}{ }^{30}$ For example, in a large prospective population-based study $(\mathrm{n}=1739)$ with 10 years of follow-up, each unit increase in positive emotion resulted in a $22 \%$ lower incidence rate of coronary heart disease, independent of demographic factors, traditional risk factors and negative emotion. ${ }^{31}$ A 2012 systematic review by Boehm and Kubzansky further documents the positive association across psychological wellbeing domains (eg, positive affect, life satisfaction, life purpose, optimism) and CVH when looking at indicators such as atherosclerosis, inflammatory markers (eg, interleukin-6, C-reactive protein), autonomic cardiac control, heart rate variability and traditional CVD risk factors. ${ }^{11}$ A majority of these studies are mindful to control for depressive symptoms and related markers of negative emotion to test the independent and clinically relevant contribution made by positive psychological attributes. Given independent contributions of psychological wellbeing to cardiac health, over and above those conferred by negative emotion and traditional risk factors, psychological assets may serve as a novel therapeutic target to improve CVH in Hispanic/Latino adults who are experiencing rising health disparities.

Although the well-being indicator of optimism has received much attention in the field of cardiovascular epidemiology in the last decade, inconsistent findings remain evident. Some studies document a cardioprotective influence ${ }^{14}{ }^{32}$ while others report null findings. ${ }^{33}$ As speculated by Kruse and Sweeny, ${ }^{34}$ it is plausible that optimism displays heterogeneous effects based on individual properties of the clinical marker under study and underlying biological context. Clinical markers most closely interconnected with stress physiology and dependent on patient-led self-care behaviour may be particularly susceptible to the influences of positive emotion. Despite divergence in the literature, the current study of the HCHS/SOL cohort replicates previous findings of MESA investigators ${ }^{14}$ where high levels of optimism were found to be associated with favourable CVH. Even though a small effect size was evident, at the population level even modest differences in CVH scores can translate into a significant reduction in subsequent deaths as effects compound over time. We did, however, observe attenuation of the effect when categorising our predictor and outcome variables, potentially as a consequence of diminished statistical power as a result of loss of information through truncation of continuous measures. Nonetheless, the present study identifies a novel clinical marker associated with $\mathrm{CVH}$, independent of depressive symptoms and prevalent CHD. Replication of findings in Hispanics/Latinos shows promise for the field of health psychology and the biopsychosocial model as they elucidate determinants, prevention efforts and treatment of chronic diseases, such as CHD.

Hispanic/Latino adults are the second-fastest growing minority group in the USA and are expected to comprise $\approx 30 \%$ of the total population by $2050 . .^{35}$ Although Hispanics/Latinos experience lower CVD-related mortality, the largest population-based cohort study of diverse Hispanic/Latino adults found that three-quarters of Hispanics/Latinos (aged 18-74 years) have at least one major CVD risk factor. ${ }^{36}$ In the current study, despite heterogeneity of the HCHS/SOL Hispanic/ Latino sample in terms of country of origin, we observed homogeneity both in the degree of optimism reported and the relationship between optimism and CVH. Thus, optimism could be an important indicator of CVD risk regardless of country of origin, sex and age. Future studies will want to consider the mechanism through which optimism influences the indices used to construct the CVH score. A multipronged process is hypothesised to underlie the pathway through which psychological well-being protects against incidence and progression of subclinical and clinical disease, that is, through promotion of healthy behaviours, augmentation of restorative biological processes and increased psychosocial and coping resources. ${ }^{3738}$ For instance, in both healthy populations and those with existing chronic illness, evidence links favourable psychological well-being with enhanced exercise regularity, smoking abstinence, ${ }^{39}$ a more prudent diet $^{4041}$ and increased medication regimen adherence. Nonetheless, more research is needed to elucidate important mediators, moderators and confounders of the relationship between positive psychological wellbeing and CVH, particularly across different contexts and cultures. For example, socioeconomic status attenuated the relationship between optimism and CVH in a Finnish sample of adults, but showed minimal influence in the HCHS/SOL cohort where almost $50 \%$ of the sample reported annual income below $\$$ US20 000. ${ }^{13}$ More research is needed to determine whether true cultural and context-specific divergence is evident or whether homogeneity of socioeconomic status in the HCHS/SOL cohort contributed to differential moderation. The present study has multiple strengths. It is the first to examine the association of optimism and CVH in a large sample of diverse Hispanics/Latinos. This allowed for examination of effect modification by Hispanic/ Latino group, yielding no apparent interaction of heritage with optimism when regressed on CVH metrics. The study was also statistically powered to adjust for important confounders unique to the Hispanic/Latino population, including acculturation and nativity status. Standardised approaches were used to obtain objective measures across health factors, for example, cholesterol. Study limitations, however, should be considered when interpreting our findings. Because dietary intake and physical activity were assessed through self-report, measurement error and misclassification bias were possible. More extensive psychometric testing in a diverse sample of Hispanics/ Latinos is warranted to begin addressing the debate of whether the LOT-R is best treated as a unidimensional measure or as a scale with a two-factor structure. This would inform uncertainty of whether optimism and 
pessimism are distinct constructs with distinct mechanistic pathways that differentially impact health. As with all cross-sectional studies, there is an inability to make definitive inferences about causality. Longitudinal studies are needed to establish causality and ascertain hypotheses regarding temporality of the association. Finally, despite the potential therapeutic nature of emotional well-being, reducing the socioeconomic disadvantage should also be explored in this population given its graded association with poor health. ${ }^{42}$

Even though optimism and other indicators of emotional well-being appear to be linked with $\mathrm{CVH}$, there remains a critical scientific gap as we do not yet know whether (or how) interventions to cultivate emotional well-being may help reduce CVD risk. Recent work testing targeted inventions to boost emotional well-being has demonstrated benefits in clinical populations and settings (eg, HIV-positive patients). Moskowitz et $a l^{43}$ found that an intervention to boost positive emotion resulted in reduced viral load in patients recently diagnosed as HIV positive. A focused intervention to improve emotional well-being may hold promise as a novel therapeutic target for promotion of $\mathrm{CVH}$. On the basis of our current findings, prevention strategies through modification of emotional well-being may be a potential avenue in helping to reach AHA's goal to increase $\mathrm{CVH}$ by $20 \%$ by $2020 .{ }^{19}$ As evidence suggests that $40 \%$ of individual variance in emotional well-being is determined by intentional activities under direct human volition, ${ }^{44}$ current evidence, in conjunction with implementation of randomised trials, will further aid in determining whether successful alteration of emotional well-being favourably impacts heart health. In conclusion, the field of health psychology is shifting away from a narrow, ill-being focus on negative psychological states towards a broader view that includes psychological wellbeing as well.

\section{Author affiliations}

${ }^{1}$ School of Social Work, University of Illinois at Urbana Champaign, Urbana, Illinois, USA

${ }^{2}$ Department of Epidemiology and Biostatistics, Michigan State University, East Lansing, Michigan, USA

${ }^{3}$ Department of Healthcare Sciences, Institute of Gerontology, Wayne State University, Detroit, Michigan, USA

${ }^{4}$ Department of Medical Social Sciences, Northwestern University, Chicago, Illinois, USA

${ }^{5}$ Department of Preventive Medicine, Northwestern University, Chicago, Illinois, USA ${ }^{6}$ Department of Psychology, San Diego State University, San Diego, California, USA ${ }^{7}$ Department of Epidemiology and Population Health, Albert Einstein College of Medicine, Bronx, New York, USA

${ }^{8}$ Department of Psychology, University of Arizona, Tucson, Arizona, USA

${ }^{9}$ Center for Research, Baptist Health South Florida, Coral Gables, Florida, USA

${ }^{10}$ Department of Psychology, University of Miami, Coral Gables, Florida, USA

${ }^{11}$ Office of Institutional Effectiveness, Southwestern College, Chula Vista, California, USA

${ }^{12}$ Gillings School of Global Public Health, University of North Carolina, Chapel Hill, North Carolina, USA

${ }^{13}$ Department of Clinical Psychology, San Diego State University/University of California, San Diego, California, USA

${ }^{14}$ Institute for Minority Health Research, University of Illinois at Chicago, Chicago, Illinois, USA
Acknowledgements RH is funded by the National Heart, Lung, and Blood Institute (NHLBI) through award number 1K01HL130712-01A1. The Hispanic Community Health Study/Study of Latinos was carried out as a collaborative study supported by contracts from the National Heart, Lung, and Blood Institute (NHLBI) to the University of North Carolina (N01-HC65233), University of Miami (N01-HC65234), Albert Einstein College of Medicine (N01-HC65235), Northwestern University (N01-HC65236) and San Diego State University (N01-HC65237). The following Institutes/Centers/Offices contribute to the HCHS/SOL through a transfer of funds to the NHLBI: National Center on Minority Health and Health Disparities, the National Institute of Deafness and Other Communications Disorders, the National Institute of Dental and Craniofacial Research, the National Institute of Diabetes and Digestive and Kidney Diseases, the National Institute of Neurological Disorders and Stroke and the Office of Dietary Supplements. The authors thank the staff and participants of HCHS/SOL for their important contributions. A complete list of staff and investigators has been provided by Sorlie et al in Ann Epidemiol. 2010; 20: 642-649 and is also available on the study website .

Contributors DW and RH researched data. HMG, WT, JTM, MRC, LCG, FJP, CRI, JMR, WA, CB, SD, FG and JLM reviewed/edited the full manuscript. MLD researched data, contributed to discussion and extensively reviewed/edited the manuscript. RH wrote the manuscript.

Funding This study was funded by National Heart, Lung, and Blood Institute (1K01HL130712, N01-HC65233, N01-HC65234, N01-HC65235, N01-HC65236, N01-HC65237).

Competing interests None declared.

Patient consent Obtained.

Ethics approval IRB at each collaborating institution.

Provenance and peer review Not commissioned, externally peer reviewed.

Data sharing statement The data and computer code used for this analysis reside at UNC Chapel Hill. The HCHS/SOL fully supports data sharing with outside investigators through processes internal to the study, based on a Data and Materials Distribution Agreement (DMDA) to protect the confidentiality and privacy of the HCHS/SOL participants and their families. Alternatively, deidentified HCHS/ SOL data are publically available at BioLINCC and dbGaP for the subset of the study cohort that authorised general use of their data at the time of informed consent.

Open Access This is an Open Access article distributed in accordance with the Creative Commons Attribution Non Commercial (CC BY-NC 4.0) license, which permits others to distribute, remix, adapt, build upon this work non-commercially, and license their derivative works on different terms, provided the original work is properly cited and the use is non-commercial. See: http://creativecommons.org/ licenses/by-nc/4.0/

(c) Article author(s) (or their employer(s) unless otherwise stated in the text of the article) 2018. All rights reserved. No commercial use is permitted unless otherwise expressly granted.

\section{REFERENCES}

1. Lichtman JH, Froelicher ES, Blumenthal JA, et al. Depression as a risk factor for poor prognosis among patients with acute coronary syndrome: systematic review and recommendations: a scientific statement from the American Heart Association. Circulation 2014;129:1350-69.

2. Labarthe DR, Kubzansky LD, Boehm JK, et al. Positive cardiovascular health: a timely convergence. J Am Coll Cardiol 2016;68:860-7.

3. Labarthe DR. From cardiovascular disease to cardiovascular health: a quiet revolution? Circ Cardiovasc Qual Outcomes 2012;5:e86-92.

4. DuBois CM, Lopez OV, Beale EE, et al. Relationships between positive psychological constructs and health outcomes in patients with cardiovascular disease: a systematic review. Int J Cardiol 2015;195:265-80.

5. Dubois CM, Beach SR, Kashdan TB, et al. Positive psychological attributes and cardiac outcomes: associations, mechanisms, and interventions. Psychosomatics 2012;53:303-18.

6. Tindle HA, Chang YF, Kuller LH, et al. Optimism, cynical hostility, and incident coronary heart disease and mortality in the Women's Health Initiative. Circulation 2009;120:656-62.

7. Rasmussen HN, Scheier MF, Greenhouse JB. Optimism and physical health: a meta-analytic review. Ann Behav Med 2009;37:239-56.

8. Boehm JK, Williams DR, Rimm EB, et al. Relation between optimism and lipids in midlife. Am J Cardiol 2013;111:1425-31. 
9. Anthony EG, Kritz-Silverstein D, Barrett-Connor E. Optimism and mortality in older men and women: The rancho bernardo study. $J$ Aging Res 2016;2016:1-9.

10. Giltay EJ, Geleijnse JM, Zitman FG, et al. Dispositional optimism and all-cause and cardiovascular mortality in a prospective cohort of elderly dutch men and women. Arch Gen Psychiatry 2004;61:1126-35.

11. Boehm JK, Kubzansky LD. The heart's content: the association between positive psychological well-being and cardiovascular health. Psychol Bull 2012;138:655-91.

12. Ford ES, Greenlund KJ, Hong Y. Ideal cardiovascular health and mortality from all causes and diseases of the circulatory system among adults in the United States. Circulation 2012;125:987-95.

13. Serlachius A, Pulkki-Råback L, Elovainio $M$, et al. Is dispositional optimism or dispositional pessimism predictive of ideal cardiovascular health? The Young Finns Study. Psychol Health 2015;30:1221-39.

14. Hernandez R, Kershaw KN, Siddique J, et al. Optimism and cardiovascular health: Multi-Ethnic Study of Atherosclerosis (MESA). Health Behav Policy Rev 2015;2:62-73.

15. Pulkki-Råback L, Elovainio M, Hakulinen $\mathrm{C}$, et al. Cumulative effect of psychosocial factors in youth on ideal cardiovascular health in adulthood: the cardiovascular risk in young finns study. Circulation 2015:131:245-53.

16. Sorlie PD, Avilés-Santa LM, Wassertheil-Smoller S, et al. Design and implementation of the hispanic community health study/study of latinos. Ann Epidemiol 2010;20:629-41.

17. Gallo LC, Penedo FJ, Carnethon M, et al. The hispanic community health study/study of latinos sociocultural ancillary study: sample, design, and procedures. Ethn Dis 2014;24:77.

18. Scheier MF, Carver CS, Bridges MW. Distinguishing optimism from neuroticism (and trait anxiety, self-mastery, and self-esteem): a reevaluation of the Life Orientation Test. J Pers Soc Psychol 1994;67:1063-78.

19. Lloyd-Jones DM, Hong Y, Labarthe D, et al. Defining and setting national goals for cardiovascular health promotion and disease reduction: the American heart association's strategic impact goal through 2020 and beyond. Circulation 2010;121:586-613.

20. González HM, Tarraf W, Rodríguez CJ, et al. Cardiovascular health among diverse hispanics/latinos: hispanic community health study/ study of latinos (HCHS/SOL) results. Am Heart J 2016;176:134-44.

21. Folsom AR, Yatsuya $H$, Nettleton JA, et al. Community prevalence of ideal cardiovascular health, by the American Heart Association definition, and relationship with cardiovascular disease incidence. J Am Coll Cardiol 2011;57:1690-6.

22. Laitinen TT, et al. Ideal cardiovascular health in childhood and cardiometabolic outcomes in adulthoodclinical perspective. Circulation 2012;125:1971-8.

23. Rasmussen-Torvik LJ, Shay CM, Abramson JG, et al. Ideal cardiovascular health is inversely associated with incident cancer: the atherosclerosis risk In communities study. Circulation 2013;127:1270-5.

24. LaMonte MJ, Durstine JL, Addy CL, et al. Physical activity, physical fitness, and Framingham 10-year risk score: the cross-cultural activity participation study. J Cardiopulm Rehabil 2001;21:63-70.

25. Arredondo EM, Sotres-Alvarez D, Stoutenberg M, et al. Physical Activity Levels in U.S. latino/hispanic adults: results From the hispanic community health study/study of latinos. Am J Prev Med 2016;50:500-8.
26. Ware J, Kosinski M, Keller SD. A 12-Item Short-Form Health Survey: construction of scales and preliminary tests of reliability and validity. Med Care 1996;34:220-33.

27. Radloff LS. The CES-D scale A self-report depression scale for research in the general population. Applied psychological measurement 1977;1:385-401.

28. Lavange LM, Kalsbeek WD, Sorlie PD, et al. Sample design and cohort selection in the Hispanic Community Health Study/Study of Latinos. Ann Epidemiol 2010;20:642-9.

29. Bottai M, Cai B, McKeown RE. Logistic quantile regression for bounded outcomes. Stat Med 2010;29:309-17.

30. Pressman SD, Cohen S. Does positive affect influence health? Psychol Bull 2005;131:925-71.

31. Davidson KW, Mostofsky E, Whang W. Don't worry, be happy: positive affect and reduced 10-year incident coronary heart disease: the Canadian nova scotia health survey. Eur Heart $J$ 2010;31:1065-70.

32. Boehm JK, Peterson C, Kivimaki M, et al. A prospective study of positive psychological well-being and coronary heart disease. Health Psychol 2011;30:259-67.

33. Boehm JK, Trudel-Fitzgerald C, Kivimaki M, et al. The prospective association between positive psychological well-being and diabetes. Health Psychol 2015;34:1013-21.

34. Kruse E, Sweeny K. Comment: well-being can improve health by shaping stress appraisals. Emotion Review 2017;9.

35. Ennis S, Rios-Vargas M, Albert N. 2012. The Hispanic population: 2010: US Census Bureau.

36. Daviglus ML, Talavera GA, Avilés-Santa ML, et al. Prevalence of major cardiovascular risk factors and cardiovascular diseases among Hispanic/Latino individuals of diverse backgrounds in the United States. JAMA 2012;308:1775-84.

37. Ryff CD, Singer BH, Dienberg Love G. Positive health: connecting well-being with biology. Philos Trans $R$ Soc Lond B Biol Sci 2004;359:1383-94.

38. Sin NL, Moskowitz JT, Whooley MA. Positive affect and health behaviors across 5 years in patients with coronary heart disease: the heart and soul study. Psychosom Med 2015;77:1058-66.

39. Carvajal SC, Wiatrek DE, Evans RI, et al. Psychosocial determinants of the onset and escalation of smoking: cross-sectional and prospective findings in multiethnic middle school samples. $J$ Adolesc Health 2000;27:255-65.

40. Shepperd JA, Maroto JJ, Pbert LA. Dispositional optimism as a predictor of health changes among cardiac patients. $J$ Res Pers 1996;30:517-34.

41. Kelloniemi H, Ek E, Laitinen J. Optimism, dietary habits, body mass index and smoking among young finnish adults. Appetite 2005;45:169-76.

42. Adler NE, Boyce T, Chesney MA, et al. Socioeconomic status and health. the challenge of the gradient. Am Psychol 1994:49:15-24.

43. Moskowitz JT, Carrico AW, Duncan LG, et al. Randomized controlled trial of a positive affect intervention for people newly diagnosed with HIV. J Consult Clin Psychol 2017;85:409-23.

44. Lyubomirsky S, Sheldon KM, Schkade D. Pursuing happiness: The architecture of sustainable change. Review of General Psychology 2005;9:111-31. 